\title{
The New Zealand rugby injury and performance project. IV. Anthropometric and physical performance comparisons between positional categories of senior $\mathrm{A}$ rugby players
}

\author{
K L Quarrie, P Handcock, M J Toomey, A E Waller
}

\begin{abstract}
Objective-To describe the anthropometric and physical performance characteristics of a sample of senior A club rugby players and to highlight differences between the positional categories of the players within the forwards and backs. Methods-94 senior A male rugby players were assessed on a number of anthropometric and physical performance assessments. The forwards were categorised into props, hookers, locks, and loose forwards. Backs were categorised into inside, midfield, and outside backs. Categories within the forwards were compared with each other, as were the categories within the backs.
\end{abstract}

Results-The anthropometric characteristics of forwards differed significantly between positional categories. Front row forwards (props and hookers) possessed highly endo-mesomorphic somatotypes, and typically rated very low for ectomorphy. Props possessed greater body mass than hookers. Locks and loose forwards were taller than the front row forwards. In terms of physical performance fewer differences were observed. Hookers performed better than props on an aerobic assessment. Locks and loose forwards were faster than the front row forwards on a $30 \mathrm{~m}$ sprint from a running start. The inside backs were shorter and lighter than the midfield and outside backs.

Conclusions-The combination of anthropometric characteristics and physical performance attributes observed allows players to best meet the demands imposed on them by their position

(BrF Sports Med 1996;30:53-56)

Key terms: rugby; anthropometry; physical performance; fitness.

The game of rugby places specific demands on players occupying the various positional roles. ${ }^{1}$ The fact that people with a range of physiques and physical attributes are able to play in the same team makes rugby unusual when compared to many other team sports, where homogeneity of physique and physical performance attributes are common. Although forwards are often considered to be "ball winners" and the backs to be "ball users", players within these broad groups have different tasks during specific phases of the game. ${ }^{1}$ Previous descriptions of the anthropometric and physical performance profiles of rugby players have shown that forwards and backs differ in terms of their typical anthropometric and physical performance characteristics. ${ }^{2-7}$

A review of previous work on the anthropometry and physical performance characteristics of rugby players shows that few studies have attempted to analyse differences between positional groups within the forwards and the backs. Rigg and Reilly ${ }^{2}$ grouped 481 st and 2nd class players into five positional categories. Comparisons were made both between positional categories and between grades. The numbers in each positional category ranged from three to seven. Differences between positional categories were observed in terms of height and weight, but somatotype did not differ significantly between positions. These investigators suggested that work with a larger sample would help further clarify the extent of differences between positions.

The purpose of this paper is to describe the anthropometric and physical performance characteristics of a sample of senior A club rugby players and to highlight differences between the positional categories of the players within the forwards and backs. The anthropometric and physical performance characteristics of the positional categories are discussed with reference to the physical demands they face during the game.

\section{Methods}

Of the 356 rugby players who enrolled in the rugby injury and performance project (RIPP) at the beginning of the 1993 rugby season, 94 were male senior A club players. The players undertook anthropometric and physical performance assessments. Both the design and methods of the project, and the anthropometric and physical performance characteristics of the players from the other grades (grouped into forwards and backs), are described in earlier papers. ${ }^{78}$ As the differences between senior A forwards and backs were outlined in the previous paper, ${ }^{7}$ comparisons between the forwards and the backs were not undertaken for this analysis.

The anthropometric measures taken were height, body mass, neck circumference, and somatotype. The physical performance assessments completed were an aerobic shuttle test, 
a maximum vertical jump, pushups, a 30 metre sprint from a standing and from a 5 metre running start, momentum obtained during the sprint from the standing start, an agility run, and a repeated high intensity shuttle test. These methods are fully described in the previous paper. $^{7}$

The following positional categories were created for the analyses. The forwards were grouped into props $(n=13)$, hookers $(n=6)$, locks $(n=15)$, and loose forwards $(n=16)$. The halfbacks and first five-eighths were grouped as inside backs $(n=11)$, the second five-eighths and centre three-quarters as midfield backs $(n=15)$, and the wing threequarters and full backs as outside backs $(n=18)$. These positional categories were chosen because the players occupying them have similar roles in the game.

Analysis of variance (ANOvA) was used to examine whether there were significant differences between the positional categories. To control for multiple testing, Bonferroni adjustments were made, with differences between groups being accepted as statistically significant at the 0.05 level if $\mathrm{P}<0.01(0.05 / 5)$ for the anthropometric variables, and $P<0.006$ $(0.05 / 8)$ for the physical performance variables.

Where the results of the ANOVA indicated that differences between positional categories were significant, post-hoc Scheffé tests were conducted to examine where the differences lay. For the forwards, the props were compared to the hookers, and the locks to the loose forwards. The props and the hookers were then combined (front row), as were the locks and loose forwards (back-five). These two groups were then compared to each other. Among the backs, the midfield and outside backs were first compared to each other. These two categories were then grouped and compared to the inside backs.

Because of the small numbers within each positional category, this study lacks statistical power. As a result of this lack of power, rela- tively large differences between groups are not necessarily statistically significant. To provide an indication of the size of differences between groups, the effect size (ES) was calculated by taking the difference between the group means, and dividing by the root mean square error (RMSE). Although the lack of statistical power is a major limitation, the numbers within each positional category are greater than have been presented in previous work outlining player anthropometry and physical performance attributes. ${ }^{2-6}$ Hence the results may provide some indications of the typical attributes of players, and of where differences between positional categories of rugby players lie. The results should, however, be interpreted with caution. The SYSTAT $^{9}$ computer package was used for the statistical analyses.

\section{Results}

FORWARDS

A summary of the anthropometric characteristics of the players, grouped by their positional categories appears in table 1 .

In terms of anthropometric characteristics, the positional categories within the forwards differed significantly with respect to height, body mass, mesomorphy, and ectomorphy. Post-hoc contrasts showed that the back-five forwards were taller than those from the front row $(\mathrm{ES}=2 \cdot 4)$, and that the locks were taller than the loose forwards $(E S=1 \cdot 6)$. Although the differences in height and endomorphy between the props and the hookers were not statistically significant, the effect sizes were large ( 1.0 and 0.8 respectively). The props possessed greater body mass than the hookers $(E S=1 \cdot 6)$. Overall, there was no significant difference in body mass between the front row forwards and the back-five $(E S=0 \cdot 0)$. The front row forwards were more mesomorphic $(\mathrm{ES}=1.4)$, and less ectomorphic $(\mathrm{ES}=1.4)$ than the back-five forwards. Figure 1 gives an indication of the typical size and shape of the forwards.

Table 1 RIPP cohort, preseason 1993. Anthropometric characteristics of senior ' $A$ ' male rugby players

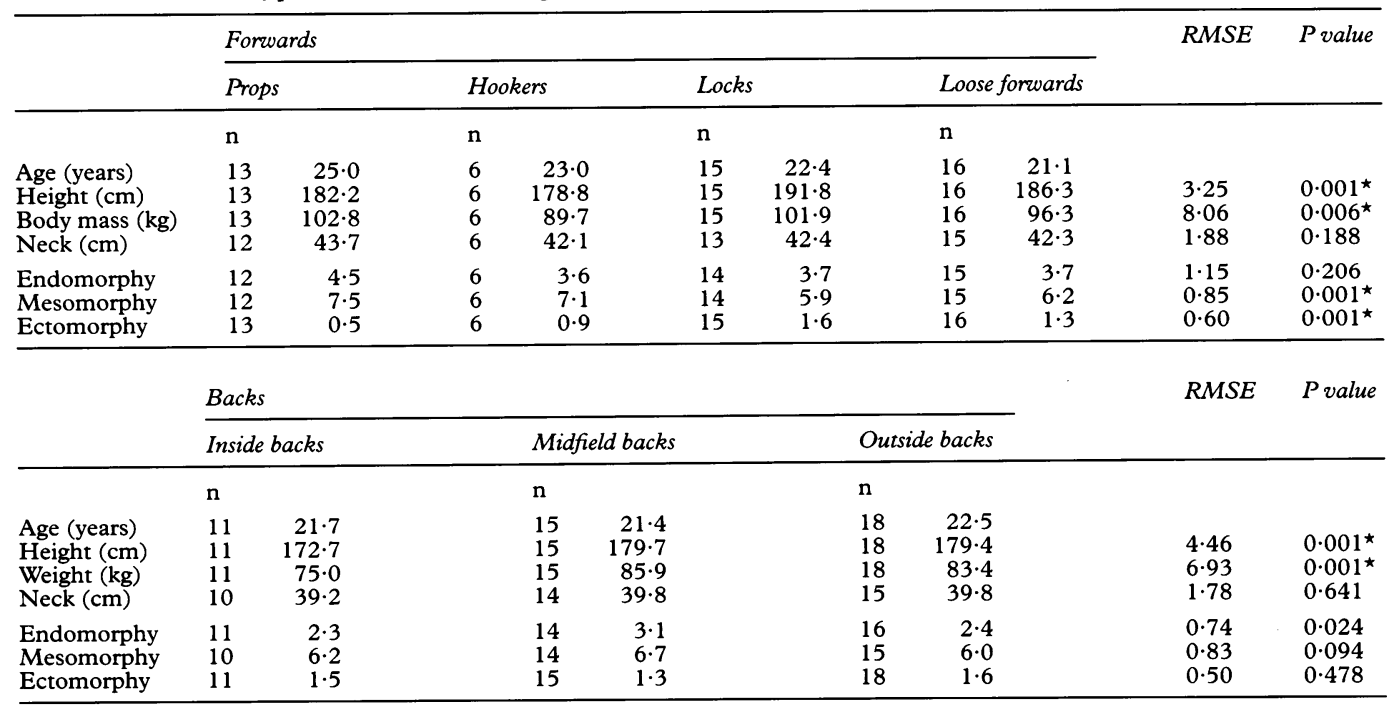

RMSE $=$ root mean square error.

$\star$ Significant at $\mathrm{P}<0.05$. 


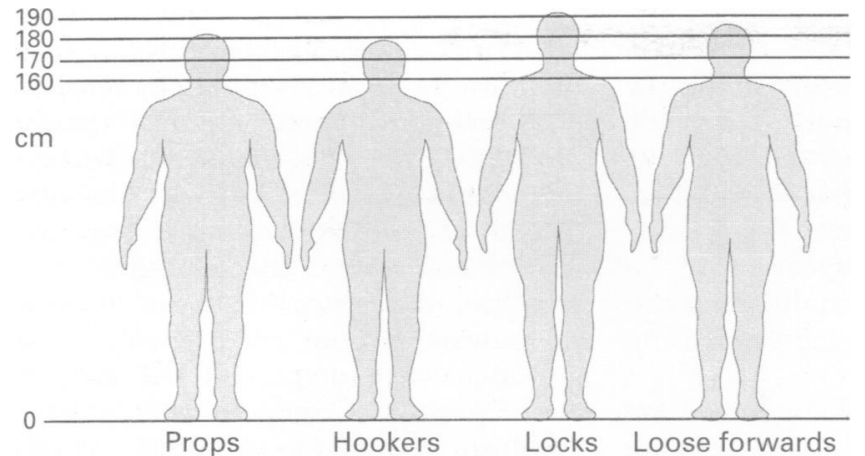

Fig 1 Rugby injury and performance project (RIPP) cohort pre-season 1993. Somatotypes of senior $A$ forwards.

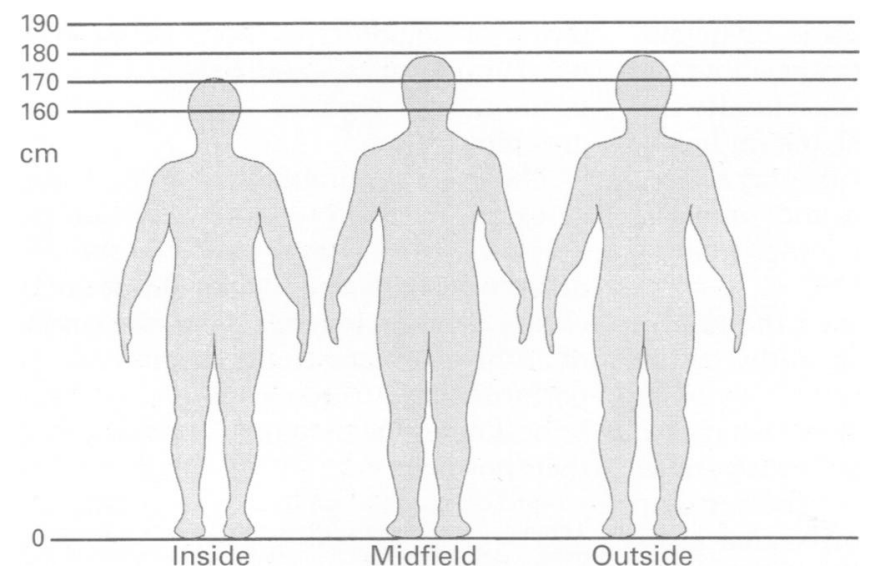

Fig 2 Rugby injury and performance project (RIPP) cohort pre-season 1993. Somatotypes of senior $A$ backs.

Significant differences between positional categories on the physical performance measures were observed on the aerobic shuttle test and the 30 metre sprint from a running start (see table 2). Post-hoc tests indicated that the hookers performed better than the props on the aerobic shuttle test $(E S=1 \cdot 8)$, and that the back-five forwards were quicker than the front row forwards on the 30-metre sprint from a running start $(E S=1 \cdot 0)$.
BACKS

The positional categories of the backs differed significantly in terms of height, body mass (see table 1), performance on the aerobic shuttle test, and momentum. Post-hoc contrasts indicated that the midfield and outside backs (grouped together) were taller $(\mathrm{ES}=1 \cdot 5)$, possessed greater body mass $(E S=1 \cdot 4)$, and obtained greater momentum during the 30 metre sprint from a standing start $(\mathrm{ES}=1 \cdot 3$ ) than the inside backs. Large effect sizes $(E S \geqslant 0 \cdot 8)$ were also observed between these groups on the aerobic shuttle test and repeated high intensity shuttle test, although in these cases the differences did not attain statistical significance. Large effect sizes were also observed between the midfield backs and the outside backs for endomorphy, mesomorphy (see fig 2), and the 30 metre sprint time from a rolling start; once again these differences did not reach statistical significance.

\section{Discussion}

The importance of possessing a certain type of physique and a typical set of physical performance characteristics to best meet the demands imposed by each of the positions in rugby is widely accepted by selectors, coaches, and players of the game. For example, The New Zealand Rugby Football Union, in their Coaching accreditation manual (level 2) state that players with ectomorphic physiques should not be placed in the front row positions. ${ }^{10}$

In the current study, the props were the heaviest, slowest, and least aerobically fit players. They possessed highly endo-mesomorphic somatotypes, and typically rated very low in terms of ectomorphy. Previous work has indicated that individuals possessing endomesomorphic somatotypes typically have better performance on strength and power measures than those with other somatotypes. ${ }^{11}$ Thus the physiques of the props probably reflect the demands placed on them for strength and

Table 2 RIPP cohort, preseason 1993. Physical performance characteristics of senior 'A' male rugby players

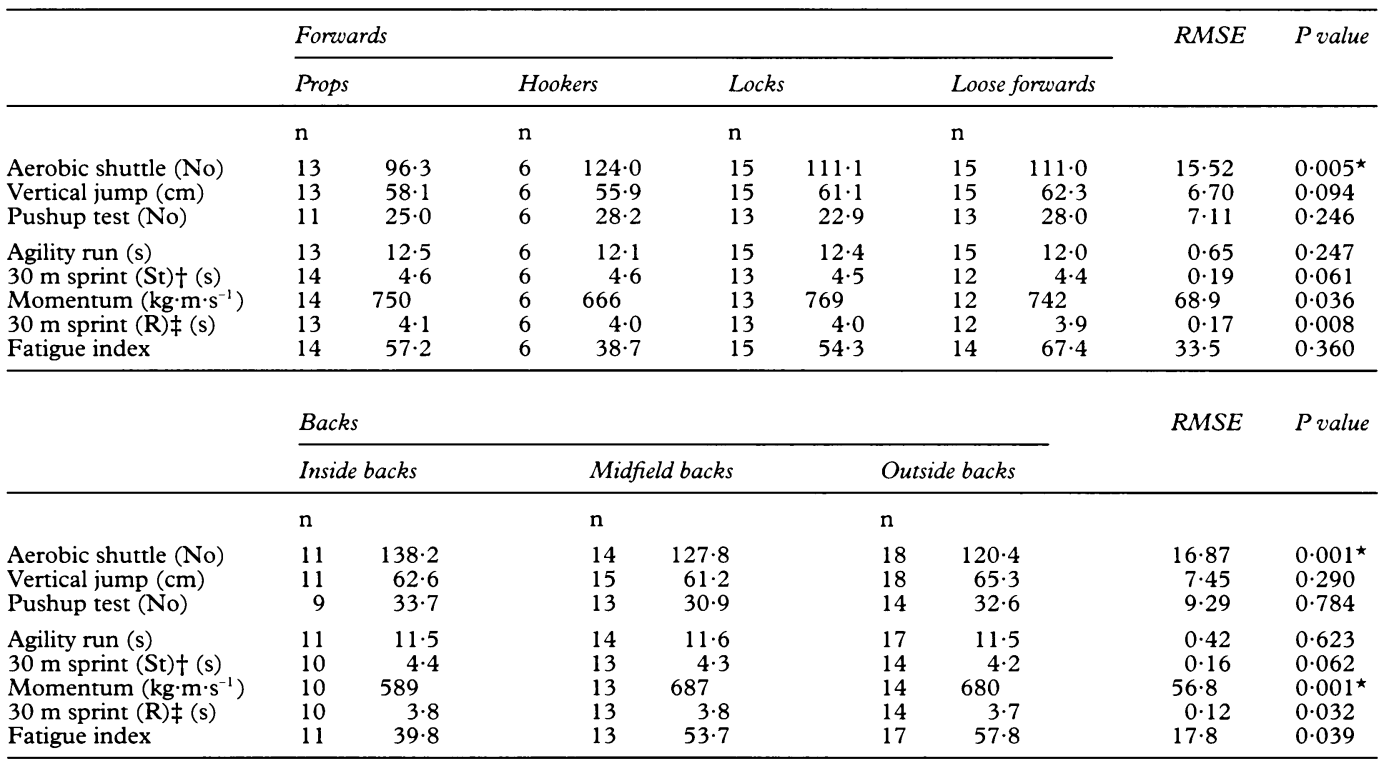

RMSE $=$ root mean square error.

$\star$ Significant at $\mathrm{P}<0.05$.

† Standing start.

Running start. 
power when competing for the ball in scrums, rucks, and mauls. The hookers were similar to the props in terms of mesomorphy, but were lighter, less endomorphic and more aerobically fit. Casual observation of the game at its highest levels would indicate that the hookers have tended towards a more "prop-like" physique over the past 15 years, as the demands for strength during scrummaging and mauls have increased with the increasing competitiveness of the game.

As the locks play a major role in contesting possession in the lineout, height and jumping proficiency are considered assets. ${ }^{12}$ Consistent with the findings of Rigg and Reilly, ${ }^{2}$ the main difference between the locks and the other positional categories was in terms of height. The jumping ability of the locks was about the same as that of the other forwards. Handcock ${ }^{12}$ has suggested that instead of relying on height to gain possession, the quality of play shown by most lineout forwards would improve from participating in specific jump training programmes.

The loose forwards are assigned the task of gaining and retaining possession of the ball in loose play, and are expected to be aggressive tacklers and to be fast over short distances. ${ }^{1}$ The loose forwards were significantly taller than the front row forwards, and shorter than the locks. Rigg and Reilly reported that loose forwards had the fitness profiles of all-round athletes. ${ }^{2}$ In the current study they did not excel on measures of physical performance when compared to the other forwards.

The players occupying different positional categories within the backs also differ in their roles in the game. The inside backs control the possession obtained by the forwards, and decide whether to launch attacking or defensive plays. In the current study the inside backs were the shortest and lightest of the positional categories. Whether the small stature of the inside backs is a result of the requirements of the position or whether the players are not selected for other positions because of lack of size is unknown.

Of the backs, the midfield backs typically have the most physical contact with the opposition. They often "crash" directly into the opposition team players at speed and, as can be seen in fig 2, were the sturdiest of the back line categories (highest in endomorphy and mesomorphy).

The outside backs are often thought of as the "speedsters" of the team, and are required to be able to beat opposition players with either pace or a combination of pace and physical strength. ${ }^{1}$

Although the outside backs were slightly faster than the other backs on the sprints, the differences between the positional categories were not statistically significant.
The present results confirm the observations of Rigg and Reilly that players occupying the various positions differ in terms of their anthropometric and physical performance attributes, though some of the patterns noted by those investigators were not observed in the present sample. For example, Rigg and Reilly reported that the most noticeable anthropometric differences between forwards and backs were in body size (height and weight), and that somatotype differed little between either positional categories or grades of play. As illustrated in figs 1 and 2, although the heights and weights of players in the present study differed between positional categories, significant differences were also observed in terms of somatotype, both between the forwards and backs, ${ }^{7}$ and between the narrower positional categories within these broad groupings.

The greater numbers within each positional category in the present study compared to previous work have enabled some of the differences between positional roles to be more clearly defined. It appears that the combination of anthropometric characteristics and physical performance attributes observed allows players to best meet the demands imposed on them by their position.

The rugby injury and performance project is funded by a grant from the Accident Rehabilitation and Compensation Insurance Corporation (ACC). The Injury Prevention Research Unit is jointly funded by the Health Research Council of New Zealand and the ACC. The views expressed in this paper are those of the authors and do not necessarily reflect those of the above organisations. The authors wish to thank the following people: organisations. The authors wish to thank the following people:
the RIPP cohort members, Ms Sheila Williams and Mr Stephen Marshall for statistical advice, and Mr Peter Scott for his Marshall for statistical advice, and Mr Peter Scott for his
assistance with graphics. We also wish to acknowledge the assistance with graphics. We also wish to acknowledge the
support and assistance of the Otago Rugby Football Union and the New Zealand Rugby Football Union.

1 Vodanovich I, Coates P, eds. New Zealand rugby skills and tactics. Auckland, New Zealand: Landsdowne Press, 1982.

2 Rigg P, Reilly T. A fitness profile and anthropometric analysis of first and second class rugby union players. In: Reilly T, Lees A, Davids K, Murphy WJ, eds. Science and Reilly T, Lees A, Davids K, Murphy WJ,

3 Maud PJ, Shultz BB. The US National rugby team: a physiological and anthropometric assessment. Physician Sportsmed 1984;12:86-94.

4 Bell W. Body composition of rugby union football players. Br $\mathcal{F}$ Sports Med 1979;13:19-23.

5 Boennec P, Prevost M, Ginet L. Somatotype de sportif de haut niveau. Résultats dans huit disciplines différentes. Med Sport 1980;54:309-18.

6 Casagrande G, Viviani F. Somatotype of Italian rugby players. I Sports Med Phys Fitness 1993;33:65-9.

7 Quarrie KL, Handcock P, Waller AE, Chalmers DJ, Toomey MJ, Wilson BD. The New Zealand Rugby Injury and Performance Project. III. Anthropometric and physical performance characteristics of New Zealand Rugby Players. Br f Sports Med 1995;29:000-000

8 Waller AE, Feehan M, Marshall SW, Chalmers DJ. The Rugby Injury and Performance Project. I. Design and methodology of a prospective follow-up study. $\mathrm{Br} \Im \mathrm{Sports}$ Med 1994;28:223-8.

9 SYSTAT for the Macintosh, Version 5.2. Evanston, Illinois: SYSTAT Inc, 1992.

10 Guy RA, Gentry SE, Stewart JJ, Smith LD, eds. New Zealand Rugby Football Union (Inc). Coaching accreditation

11 Bale P, Colley E, Mayhew J. Size and somatotype correlates of strength and physiological performance in adult male of strength and physiological performance
students. Aust f Sci Med Sport 1984;16:2-6.

12 Handcock P. Physical preparation for rugby union. $f$ Phys Educ NZ 1993;26:7-9. 